\title{
Efeito da gravidez na adolescência sobre os resultados perinatais em maternidades de nivel terciário no ano de 2003 no estado do Ceará - Brasil
}

Effect of pregnancy in adolescence on perinatal results in tertiary level maternities in 2003 in the state of Ceará - Brazil

Autora: Silvia de Melo Cunha

Orientadora: Profa. Dra. Zenilda Vieira Bruno

Dissertação apresentada ao Departamento de Saúde Materno-Infantil (Mestrado em Tocoginecologia) Universidade Federal do Ceará, em 15 de dezembro de 2006.

Objetivos: avaliar de forma comparativa peso ao nascimento, idade gestacional e internamento de recémnascido (RN) em unidade de terapia intensiva (UTI), em três grupos de mães, divididos em adolescentes precoce (11-15 anos), tardia (16-19 anos) e não adolescentes (>19 anos). Métodos: estudo observacional, descritivo, transversal, que estuda a relação da idade materna com idade gestacional e peso ao nascer de $\mathrm{RN}$ internados na UTI. Aplicados os testes qui-quadrado de Pearson, relação linear, exato de Fisher e calculado adds ratio (OR). Resultados: embora exista indicativo de relação linear, $\mathrm{p}=0,097(<0,100)$, onde à medida que aumenta a idade da mãe, diminui a incidência de RN em UTI, não se obteve estatisticamente, associação entre idade materna e internamento em UTI. Existe, porém, associação estatística entre idade gestacional e idade materna e entre a primeira e peso do $R N$ ao nascer $(p<0,001)$. Observou-se maior percentual de prematuridade, baixo peso e muito baixo peso ao nascer nos RN de adolescentes precoces, quando comparado com adolescentes tardias e não adolescentes. Conclusões: não foi encontrada associação significativa entre a idade materna e o internamento do RN em UTI. Existe diferença estatisticamente significante entre adolescentes e não adolescentes, quanto ao percentual de prematuridade, baixo peso e muito baixo peso ao nascer, mas não foi encontrada significância estatística, quando comparadas adolescentes precoces e tardias.

PALAVRAS-CHAVE: Gravidez na adolescência; Prematuridade; Baixo peso ao nascer

Resumo de Tese

\section{Misoprostol sublingual versus vaginal para indução do parto Sublingual versus vaginal misoprostol for induction of labor}

Autor - Francisco Edson de Lucena Feitosa

Orientador - Prof.Dr. Renato Passini Júnior

Tese de Doutorado apresentada ao Curso de Pós-Graduação em Tocoginecologia da Faculdade de Ciências Médicas da Universidade Estadual de Campinas, em 12 de dezembro de 2005.

Objetivo: comparar a eficácia e segurança da utilização do misoprostol sublingual versus vaginal na indução do parto a termo. Métodos: realizou-se um ensaio clínico controlado, randomizado e duplo-cego, no período de $1^{\circ}$. de junho de 2004 a 30 de março de 2005. Para atingir diferença mínima entre os grupos de $20 \%$, erro alfa de $5 \%$ e um poder $(1-\S)$ de $80 \%$, foram randomizadas 150 gestantes, das quais 75 receberam $25 \mathrm{mcg}$ de misoprostol sublingual e placebo vaginal a cada 6 horas e 75 receberam $25 \mathrm{mcg}$ de misoprostol vaginal e placebo sublingual a cada 6 horas. O desfecho primário avaliado foi freqüência de parto vaginal. A análise estatística utilizou o teste qui-quadrado de associação, teste exato de Fisher e de Mann-Whitney, calculando-se a razão de risco com intervalo de confiança de 95\%. Utilizou-se nível de significância de 5\%.

Resultados: os grupos do misoprostol sublingual e vaginal foram similares e comparáveis. A proporção de partos vaginais foi de $57,3 \%$ no grupo sublingual e $69,3 \%$ no grupo vaginal $(R R=1,2 ;$ IC9 $5 \%=0,8-1,8)$. A média de intervalo de tempo entre a indução e o parto foi de $18 \mathrm{~h}$ e 48 minutos no grupo sublingual (SL) e $18 \mathrm{~h}$ e 15 minutos no grupo vaginal (V). Não houve diferença significante na freqüência de partos vaginais após uma única dose de misoprostol, no número de gestantes que necessitaram de quatro doses de misoprostol, na freqüência de falha de indução, na necessidade de utilização de ocitocinan, na freqüência de taquissistolia, na presença de mecônio intraparto e nos resultados neonatais.

Conclusão: o misoprostol sublingual $(25 \mathrm{mcg}$ a cada 6 horas) foi tão eficaz e seguro quanto à mesma dose do misoprostol vaginal para indução do parto.

PALAVRAS-CHAVE: Misoprostol; Trabalho de parto; Trabalho de parto induzido; prostaglandinas 